Proc. Indian Acad. Sci. (Chem. Sci.). Vol. 103, No. 1, January 1991, pp. 77-82

(i) Printed in India.

\title{
Relaxation dominated by inertia: Solvation dynamics of a small ion in a dipolar solvent
}

\author{
A CHANDRA and B BAGCHI* \\ Solid State and Structural Chemistry Unit, Indian Institute of Science, Bangalore 560012 , \\ India \\ MS received 4 December 1990
}

\begin{abstract}
It is shown from an analytical theory that the solvation dynamics of a small ion can be controlled largely by the inertial response of the dipolar solvent when the liquid is in the underdamped limit. It is also shown that this inertial response arises primarily from the long wavelength (with wavevector $k \simeq 0$ ) processes which have a collective excitation-like behaviour. The long time decay is dominated by the processes occurring at molecular lengthscales. The theoretical results are in good agreement with recent computer simulation results.
\end{abstract}

Keywords. Relaxation; inertia; solvation dynamics of small ions; dipolar solvent.

The dynamics of solvation of polar molecules (ionic, dipolar or quadrupolar) in dipolar liquids is a subject of tremendous current interest (Bagchi 1989; Barbara and Jarzeba 1990; Maroncelli et al 1989; Fleming and Wolynes 1990). Thanks to the intense experimental and theoretical activity in this field in the last five years or so, an understanding of this complex phenomenon is beginning to emerge. It has become clear that the continuum model based theories (Bagchi et al 1984; van der Zwan and Hynes 1985) are inadequate to describe the complexities of the solvation phenomena. Computer simulations (Maroncelli and Fleming 1988; Karim et al 1988) have revealed many molecular aspects of solvation. A large number of microscopic studies (Calef and Wolynes 1983; Wolynes 1987; Loring and Mukamel 1987; Friedrich and Kivelson 1987; Nichols and Calef 1988; Bagchi and Chandra 1988, 1989; Chandra and Bagchi 1988, 1989, 1990a; Raineri et al 1990; Wei and Patey 1990; Fried and Mukamel 1990) have been carried out to understand solvation dynamics. Impressive progress has been made in experimental studies also. The experimental results from different groups have been reviewed by Barbara and Jarzeba (1988, 1990), Simon (1988) and by Maroncelli et al (1989). All these studies show that molecular aspects of relaxation are important and that the solvation dynamics is nonexponential in general. It has also been pointed out that the translational modes of the solvent can be very important in solvation dynamics (Chandra and Bagchi 1988, 1989; Bagchi and Chandra 1989). Chandra and Bagchi (1990b, 1991) have also studied the possible role of the solvent inertial response. Very recently, Maroncelli (1991) has performed computer simulations

\footnotetext{
* For correspordence
} 
of solvation dynamics in acetonitrile to find that this inertial response can indeed be very important in the solvation dynamics of small ions.

Another interesting aspect of the computed relaxation is the existence of a persistent oscillation with frequency approximately equal to $3.5 \times 10^{13} \mathrm{~s}^{-1}$. This is clearly a rather large frequency at which the frequency dependence of the friction may be important.

In this communication we show that these inertial effects can be explained and understood from our earlier theory with inertial response (Chandra and Bagchi 1990b, 1991a). Several interesting conclusions also emerge from this study. We find a good agreement with some aspects of the computer simulation results of Maroncelli (1991). Although our results show oscillations at a frequency very close to the observed one, the persistent oscillations observed in computer simulations are not observed. We discuss the possible reasons for this.

As before (Bagchi 1989), the time dependent solvation energy is assumed to be given by the following expression in the Fourier space (with $k$ as the Fourier variable)

$$
E_{\mathrm{solv}}(t)=-\frac{1}{2}(2 \pi)^{-3} \int \mathrm{dk} E_{0}(k) P_{L}(k, t),
$$

where $P_{L}(k, t)$ is the time dependent longitudinal polarization of the solvent. The Fourier transform of the bare electric field of the ion, $E_{0}(k)$, is given by the following expression

$$
E_{0}(k)=\frac{4 \pi i q}{k} \frac{\sin k r_{c}}{k r_{c}},
$$

where $q$ is the charge of the ion, $r_{c}=a+\sigma / 2$, where $a$ is the radius of the solute ion and $\sigma$ is the diameter of a solvent molecule. In deriving (2), we have assumed that the solute ion can be represented by a rigid sphere of radius $a$ with a point charge $q$ at the centre. It was shown earlier (Chandra 1990; Chandra and Bagchi 1990b; Bagchi and Chandra 1991) that $P_{L}(k, t)$ can be given by the following equation of motion when the inertial response of the solvent molecules are included

$$
\begin{aligned}
\frac{\partial}{\partial t} P_{L}(k, t)= & -A B k^{2} f_{L}(k) \int_{0}^{t} \mathrm{~d} t^{\prime} P_{L}\left(k, t^{\prime}\right) e^{-A B\left(t-t^{\prime}\right) / 2 p^{\prime}} \\
& -2 B f_{L}(k) \int_{0}^{t} \mathrm{~d} t^{\prime} P_{L}\left(k, t^{\prime}\right) e^{-B\left(t-t^{\prime}\right)}
\end{aligned}
$$

where the function $f_{L}(k)$ and the other parameters are given by

$$
\begin{aligned}
f_{L}(k) & =1-\frac{\rho_{0}}{4 \pi} C(110 ; k) \\
A & =I / m \sigma^{2} \\
B & =k_{B} T / I D_{R}^{2} \\
p^{\prime} & =D_{T} / 2 D_{R} \sigma^{2}
\end{aligned}
$$

where $C(110 ; k)$ is the $(110)$ component of the spherical harmonic expansion of the 
two-particle direct correlation function of the dipolar liquid (Gray and Gubbins 1984). It is a measure of the intermolecular interaction among the dipolar solvent molecules. In fact, it is related to the force constant of the longitudinal polarization fluctuations (Chandra and Bagchi 1991b). $m, I, D_{R}$ and $D_{T}$ are the mass, the moment of inertia and the rotational and the translational diffusion coefficients of the solvent molecules, respectively. $p^{\prime}$, defined by (7), is a relative measure of the importance of the translational modes of the liquid in the solvation dynamics. For the derivation of (3), we refer to a recent publication (Chandra and Bagchi 1990b).

Equation (3) is essentially a third order differential equation. The parameters $A$ and $B$ gauge the importance of the inertial effects. For a given solvent, $A$ is fixed, but $B$ can be varied by varying temperature, pressure, viscosity etc. Inertial response is important when $B$ is small $(B<50)$. $p^{\prime}$ also plays an important role, especially at intermediate wavevectors (for $k>2 \pi / \sigma$ ). We find that in the solvation of small ions, these modes are very important. In the inertial limit, they can give rise to pronounced oscillations. As mentioned earlier, such oscillations have already been observed in the computer simulations of Maroncelli (1991).

Equation (3) can be solved by Laplace transforming it to the frequency domain (with $z$ as the complex frequency) and performing the complex integrals to recover the time dependent behaviour.

with

$$
P_{L}(k, t)=P_{L}(k, t=0) g_{L}(k, t),
$$

$$
g_{L}(k, t)=\sum_{i=1}^{3} A_{i}(k) e^{z_{i}(k) t},
$$

where $z_{i}$ are the three roots of the algebric equation obtained from (3) by Laplace transformation and $A_{i}(k)$ 's are the respective weight factors. The steps are straightforward and details are available elsewhere (Chandra and Bagchi 1990b, 1991 a; Bagchi and Chandra 1991). It suffices to say here that the roots $z_{i}(k)$ can be complex depending on the values of $k, p^{\prime}, A$ and $B$. When $z_{i}(k)$ is complex with a very small real part, it is fair to conclude that the longitudinal fluctuation with wavenumber $k$ is a collective excitation, known as dipolaron (Chandra and Bagchi 1990b).

We next present the numerical results. We consider the same system as simulated by Marconcelli (1991). The solvent is acetonitrile with the following simulated values. Static dielectric constant $\left(\varepsilon_{0}\right)=33$, Debye relaxation time $\left(\tau_{D}\right)=4 \cdot 1$ ps. The solutesolvent size ratio is 0.67 . We find $D_{R}$ from $\tau_{D}$ by using the linearized theory (Chandra and Bagchi 1989) which gives the following relation between the two

$$
\tau_{D}=\left[2 D_{R}\left\{1+\frac{\rho_{0}}{4 \pi} C(111 ; k=0)\right\}\right]^{-1},
$$

where $C(111 ; k)$ is the $(111)$ th component in the spherical harmonic expansion of the two particle direct correlation function.

We have used the mean spherical approximation (MSA) to evaluate the direct correlation function component. Equation (10) gives $D_{R}=0.415 \mathrm{ps}^{-1}$ which gives $B=29$ for the present system. The value of $D_{T}$ poses a problem. If we use the experimentally measured values of $D_{T}$, then we get $p^{\prime}=0-0233$. However, one should really calculate $D_{T}$ from computer simulations, which, unfortunately, are not available. Maroncelli (1991) uses a value $p^{\prime}=0.07$. Because of the uncertainty, we chose a value 
$p^{\prime}=0.05$ which should be qualitatively correct. The value of the parameter $A$ is 0.06 .

The results in solvation dynamics are usually expressed in terms of a solvation time correlation function, defined by

$$
C_{s}(t)=\frac{E_{\text {solv }}(t)-E_{\text {solv }}(t=\infty)}{E_{\text {solv }}(t=0)-E_{\text {solv }}(t=\infty)},
$$

where $E_{\text {solv }}(t)$ is the solvation energy at time $t$.

The results of our numerical calculations are shown in figure 1 where $C_{s}(t)$ is plotted against time. It can be seen from this figure that in the present case the inertial response is very important and the overdamped limit does not provide the correct description. A large portion of the decay occurs in the inertial regime. There is an oscillation around $t \simeq 4 \mathrm{ps}$ which is followed by a smooth, almost exponential decay. This bimodal nature of $C_{s}(t)$ was also noted by van der Zwan and Hynes (1985).

The short time behaviour of the calculated $C_{s}(t)$ is in good agreement with the computer simulation results (Maroncelli 1991). However, the persistent, high frequency oscillations in the long time are absent here. In order to understand the origin of this high frequency oscillation, we investigated the nature of the roots $\left(z_{i}\right)$ of the polarization modes and the values of the three roots are given in table 1 for two different wavevectors, $\mathrm{k} \sigma=0$ and $\mathrm{k} \sigma=6$. The table shows that the $k=0$ mode is actually a short lived collective excitation, but the intermediate wavevector mode is always damped. The most interesting point here is that the frequency of oscillation of the $k=0$ mode is $4.5 \times 10^{13} \mathrm{~s}^{-1}$ which is very close to the oscillation frequency

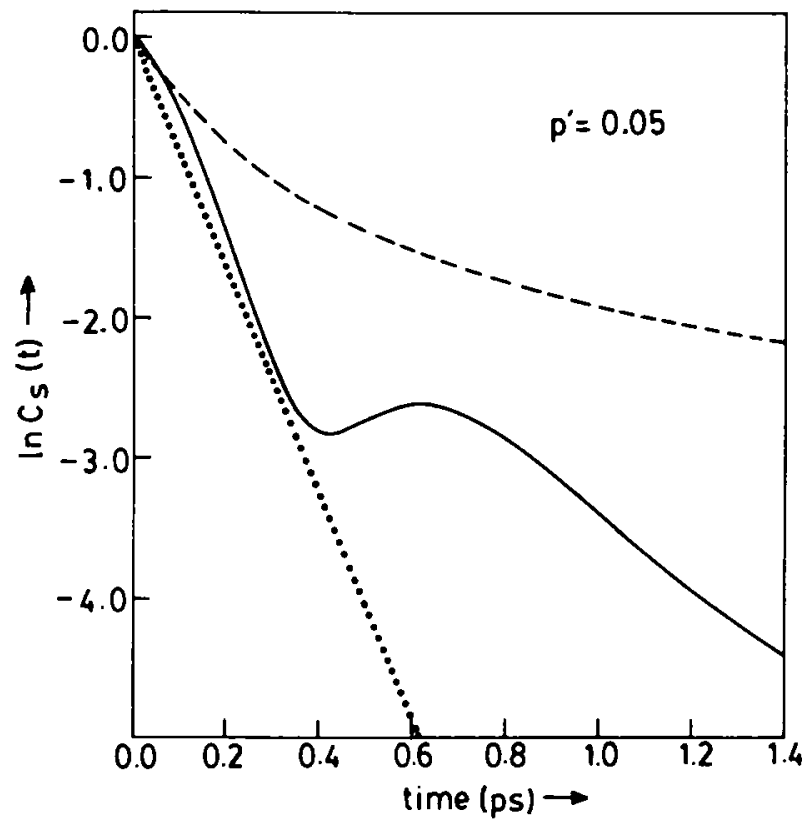

Figure 1. The decay of the solvation time correlation function, $C_{\mathrm{a}}(t)$ [defined by $\left.(11)\right]$, is plotted against time for a monatomic ion in the solvent acetonitrile with $\varepsilon_{0}=33, \varepsilon_{\infty}=1 \cdot 0$, $\tau_{D}=4 \cdot 1 \mathrm{ps}, p^{\prime}=0.05, B=29.0$ and $A=0-06$. The solute-solvent size ratio is 0.67 . The solid line is the calculated decay with inertia, the dashed line shows the decay in the overdamped limit and the dotted line shows that continuum limit [with relaxation time $\left(\varepsilon_{\infty} / \varepsilon_{0}\right) \tau_{p}$ ]. 
Table 1. The real and the imaginary components of the three roots $\left(z_{i}\right)$ of the polynomial for the decay of the polarization in the complex plane at two different wavevectors.

\begin{tabular}{lcc}
\hline $\begin{array}{c}\text { Wavevector of } \\
\text { the mode }(k \sigma)\end{array}$ & $\begin{array}{c}\text { Real part } \\
\text { (in } 10^{13} \mathrm{~s}^{-1} \text { ) }\end{array}$ & $\begin{array}{c}\text { Imaginary part } \\
\text { (in } 10^{13} \mathrm{~s}^{-1} \text { ) }\end{array}$ \\
\hline & -4.04 & 0.0 \\
0.0 & -3.37 & 4.52 \\
& -3.37 & -4.52 \\
6.0 & -6.26 & 0.0 \\
& -1.32 & 0.0 \\
& -3.03 & 0.0 \\
\hline
\end{tabular}

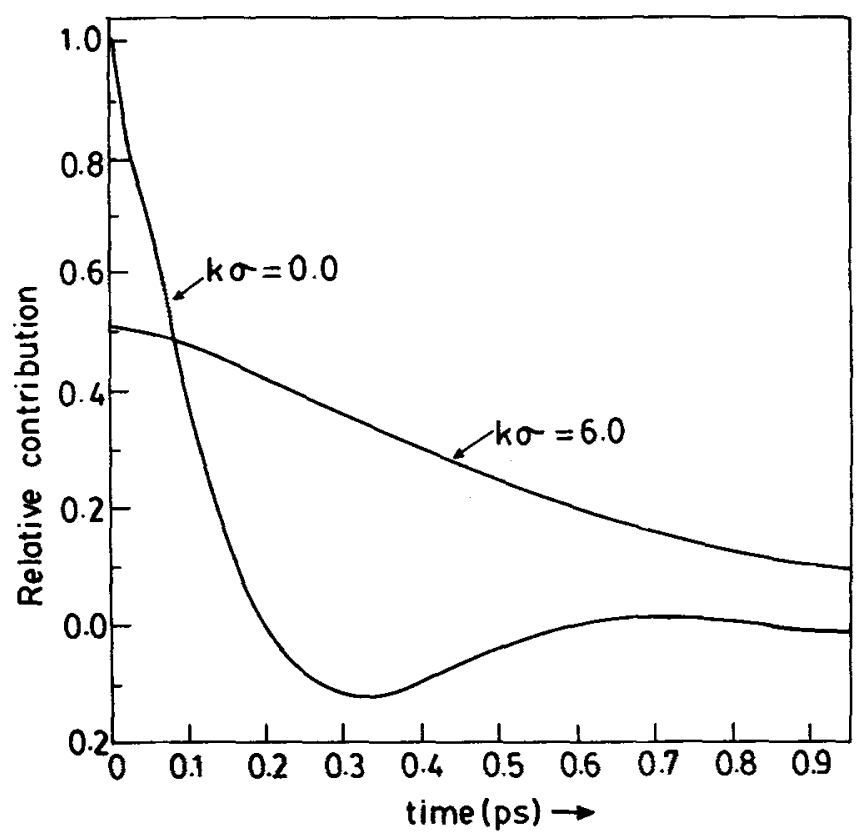

Figure 2. The decomposition of the relaxation behaviour into the long wavelength $(k \sigma=0)$ and the intermediate wavelength $(k \sigma=6)$ contributions. The initial values indicate the relative initial wights calculated within an interval of 0.05 around the modes.

(approximately $3.5 \times 10^{13} \mathrm{~s}^{-1}$ ) observed in the computer simulations. This has led us to conclude that the oscillations observed in the computer simulations of Maroncelli (1991) are the manifestations of the coupling of the ionic field with the solvent collective excitations.

Also note that the damped decay of the intermediate wavevector $(k \sigma=6)$ mode is responsible for the slow decay at the long time. It is really interesting to find such a decomposition of the decay behaviour. These very different decay profiles are shown in figure 2 where the relative $E_{\text {solv }}(t)$ from the two modes $(k \sigma=0$ and $k \sigma=6)$ are plotted for comparison. Numerical calculations also show that the relative contributions of 
the $k \sigma=0$ and $k \sigma=6$ modes are approximately $66 \%$ and $34 \%$, respectively. This is in good agreement with the simulation results of 80 and $20 \%$ contributions of the inertial and the damped modes, respectively.

We should note here that the harmonic oscillator model of van der Zwan and Hynes (1985) can also show a behaviour not too dissimilar from the computer simulation results, but the vdZH model is a continuum theory based model and hence considers only the $k=0$ mode. Therefore, they can explain the initial inertial response and also the oscillations, but they fail to recover the slow longtime decay which is shown here to arise from the intermediate wavevector (or molecular length scale) processes.

In view of the results presented here, it is fair to conclude that many aspects of the simulation results of Maroncelli (1991) on the solvation of a small monatomic ion in acetonitrile can be explained from the existing theory. We shall discuss in a later publication that the $k \sigma=0$ collective excitation may exist longer to produce the observed behaviour if the viscoelastic response of the solvent is included in the hydrodynamic description.

\section{References}

Bagchi B 1989 Annu. Rev. Phys. Chem. 40115

Bagchi B and Chandra A 1988 Proc. Indian Acad. Sci. (Chem. Sci.) 100353

Bagchi B and Chandra A 1989a Chem. Phys. Lett. 155533

Bagchi B and Chandra A 1989b J. Chem. Phys. 907338

Bagchi B and Chandra A 1991 Adv. Chem. Phys. 801

Bagchi B, Oxtoby D W and Fleming G R 1984 Chem. Phys. 86257

Barbara P F and Jarzeba W 1988 Acc. Chem. Rev. 21195

Barbara P F and Jarzeba W 1990 Adv. Photochem. 151

Chandra A 1990 Theoretical studies on collective orientational relaxation, solvation dynamics and electron transfer reactions in dense dipolar liquids, $\mathrm{PhD}$ thesis, Indian Institute of Science, Bangalore

Chandra A and Bagchi B 1988 Chem. Phys. Lett. 15147

Chandra A and Bagchi B 1989a J. Phys. Chem. 936996

Chandra A and Bagchi B 1989b J. Chem. Phys. 912594

Chandra A and Bagchi B 1990a J. Phys. Chem. 943152

Chandra A and Bagchi B 1990b J. Chem. Phys. 926833

Chandra A and Bagchi B 199ia J. Chem. Phys. (in press)

Chandra A and Bagchi B 1991b J. Chem. Phys. (in press)

Calef D F and Wolynes P G 1983 J. Chem. Phys. 784145

Fleming G R and Wolynes P G 1990 Phys. Today (May) 36

Fried I E and Mukamel S $1990 \mathrm{~J}$. Chem. Phys. 93932

Friedrich V and Kivelson D 1987 J. Chem. Phys. 866425

Grey C G and Gubbins K E 1984 Theory of molecular fluids (Oxford: Clarendon) vol. 1

Karim O, Haymet A D J, Banet M J and Simon J D.1988 J. Phys. Chem. 923391

Loring R F and Mukamel S 1987 J. Chem. Phys. 871272

Maroncelli M $1991 \mathrm{~J}$. Chem. Phys. (in press)

Maroncelli M and Fleming G R 1988 J. Chem. Phys. 895044

Maroncelli M, McInnis J and Fleming G R 1989 Science 2431674

Nichols A III and Calef D F $1988 \mathrm{~J}$. Chem. Phys. 893783

Raineri F, Zhou Y, Friedman H L and Stell G 1991 Chem. Phys. (in press)

Simon J D 1988 Aco. Chem. Res. 21128

van der Zwan G and Hynes J T 1985 J. Phys. Chem. 894181

Wei D and Patey G N 1990 J. Chem. Phys. 931399

Wolynes P G 1987 J. Chem. Phys. 865133 